\title{
SOPORTE VENTILATORIO NO INVASIVO EN INSUFICIENCIA VENTILATORIA AGUDA SEVERA EN ADOLESCENTES CON ENFERMEDAD NEUROMUSCULAR Y OBESIDAD. ESTUDIO DE CASOS NON-INVASIVE VENTILATORY SUPPORT IN SEVERE ACUTE VENTILATORY FAILURE IN ADOLESCENTS WITH NEUROMUSCULAR DISEASE AND OBESITY. STUDY OF CASES
}

\author{
Dr. Francisco Prado A., ${ }^{1,2}$; Dr. Carlos Valdebenito P. ${ }^{1,3}$.; KIgo. Italo Sassarini C.4; KIgo. Pedro Morales C.5; \\ Dra. Valeska Madrid SM'; Dra. Carolina Barrientos M. ${ }^{1}$. \\ 1. Residente de pediatría y Hospitalización Domiciliaria Pediátrica, Hospital Clínico San Borja Arriarán. Santiago, Chile. \\ 2. Pediatra Broncopulmonar, Hospital Clínico San Borja Arriarán. Departamento de Pediatría y Cirugía Infantil, Campus Centro, \\ Universidad de Chile. Santiago, Chile. \\ 3. Departamento de Pediatría y Cirugía Infantil, Campus Centro, Universidad de Chile Santiago, Chile. \\ 4. Kinesiólogo Hospital Clínico San Borja Arriaran, Chile. \\ 5. Kinesiólogo Unidad Hospitalización Pediátrica, Hospital Clínico San Borja Arriaran, Chile.
}

\section{ABSTRACT}

Noninvasive ventilatory support (NIVS) combined with mechanical cough assist (MI-E) is an effective tool to treat patients with acute ventilatory failure due to neuromuscular disorders (NMD). Airway respiratory infection could be lethal or with risk of endotraqueal intubation, especially when vital capacity (VC) is less than $15 \mathrm{ml} / \mathrm{k}$. We report 2 obese adolescents, aged 11 and 14 years old, with myasthenic crisis (MC) and Duchenne muscular dystrophy (DMD). The last one with a severe cifoescoliosis treated with nocturnal noninvasive ventilation at home. MC girl has been treated with pyridostigmine, prednisolone and mycophenolate. They were admitted for thymectomy and spinal surgery arthrodesis respectively. After admission they developed airway respiratory infection triggering by Methaneumovirus and were treated with oxygen therapy, non-invasive ventilation with low-pressure support and EV immunoglobulin for the MC girl. After 48 $\mathrm{h}$ both patients developed severe respiratory failure, Sa/FiO2 < 200, atelectasis of lower lobes and difficulty to swallow, a peak cough flow (PFT) $<100 \mathrm{l} / \mathrm{m}$ and CV $800 \mathrm{ml}(11 \mathrm{ml} / \mathrm{k})$ in the patient with CM and $200 \mathrm{ml}(2.5 \mathrm{ml} / \mathrm{k})$ in the patient with DMD.We decided switch to NIVS with Trilogy $®$ and BipapA40® equipment respectively, in S/T mode (spontaneous/time) and AVAPS (average volume assured in support pressure). Oxygen therapy was quickly stopped and intensive therapy with MI-E was started. Both patients improved clinically as well as the daily measure of VC, PFC and PEF-MI-E. Combination between NIVS and MI-E with a systematic application until SaO2 normalize to at least $95 \%$ with no oxygen therapy can avoid tracheal intubation in patients with NMD, unlike the aggravation produced by AVNI and oxygen therapy with classical criteria.

Key words: neuromuscular diseases, noninvasive ventilatory support, and mechanical in-exsufflator protocols.

RESUMEN

El soporte ventilatorio no invasivo (SVNI) y la rehabilitación respiratoria con apilamiento de aire más tos asistida manual 0 mecánica, son efectivas para tratar la insuficiencia ventilatoria aguda en pacientes con enfermedades neuromusculares (ENM) y deterioro progresivo de la bomba respiratoria. Las agudizaciones gatilladas por infecciones respiratorias causan insuficiencia ventilatoria aguda potencialmente mortal y con alto riesgo de intubación, en especial cuando la capacidad vital (CV) es $<$ de $15 \mathrm{ml} / \mathrm{k}$. Se reportan 2 adolescentes obesos con ENM de 11 y 14 años con miastenia gravis y distrofia muscular de Duchenne (DMD) con asistencia ventilatoria no invasiva nocturna con baja presión de soporte (AVNI), ingresados para timectomía y artrodesis de columna respectivamente. Una vez ingresados evolucionan con insuficiencia ventilatoria aguda secundaria a una infección respiratoria por Metaneumovirus. Inicialmente fueron manejados con oxigenoterapia, AVNI y gamaglobulina endovenosa en el caso de la paciente con crisis miasténica (CM). A las $48 \mathrm{~h}$ presentan dificultad respiratoria severa, Sa/FiO2 < 200, atelectasias bibasales y disfagia, CV de $800 \mathrm{ml}(11 \mathrm{ml} / \mathrm{k})$ en el paciente con CM y de $200 \mathrm{ml}(2,5 \mathrm{ml} / \mathrm{k})$ en el paciente con DMD y un pico flujo tosido (PFT) < 100 l/m. Se cambia a SVNI con equipo Trilogy® y BipapA40®, en modalidad S/T (espontáneo/tiempo) y AVAPS (volumen promedio asegurado en presión de soporte) con altos parámetros ventilatorios; suspendiendo rápidamente la oxigenoterapia, al combinar tos mecánicamente asistida con in-exsufflator (MI-E) en forma intensiva. Ambos pacientes presentan mejoría clínica sostenida, de la CV, PFT y pico flujo exuflado máximo con MI-E (PFE-MI-E). El SVNI más la aplicación sistemática del MI-E hasta lograr Sa02 de al menos 95\% con oxígeno ambiental evita la intubación endotraqueal en ENM, a diferencia del agravamiento producido por AVNI y oxigenoterapia con criterios clásicos.

Palabras claves: enfermedades neuromusculares, soporte ventilatorio no invasivo, protocolos de tos asistida mecánica.

Correspondencia:

Dr. Francisco Prado Atlagic

fpradoatlagic@gmail.com 


\section{INTRODUCCIÓN}

Se reconoce que el $75 \%$ de las muertes en pacientes con enfermedades neuromusculares (ENM) se deben a fallo respiratorio agudo por infecciones respiratorias agudas generalmente asociadas a atelectasias $(1,2)$. Del mismo modo los postoperatorios de cirugías de cifoescoliosis y otras cirugías electivas en estos pacientes, son situaciones de riesgo para ser traqueostomizados por fallo de la extubación, sino son apropiadamente manejados (3-8).

También es conocido que la hipoventilación asociada a ENM progresa en función de la capacidad vital (CV). La que va desde hipoventilación nocturna con requerimientos de soporte ventilatorio no invasivo (SVNI), cuando la CVF es menor al 30\% del predicho en niños menores de 10 años o menor de $1500 \mathrm{ml}$ en niños mayores de 10 años; a hipoventilación diurna con requerimiento de SVNI continuo en aquellos sin autonomía ventilatoria con $\mathrm{CV}<$ de $10 \mathrm{ml} / \mathrm{k}(1,2,3)$.

La obesidad, sobre todo cuando hay alto índice de masa corporal, no sólo se relaciona con apneas obstructivas del sueño (SAOS), sino que también con insuficiencia ventilatoria, como ocurre en el síndrome de hipoventilación y obesidad (9), en especial cuando la bomba respiratoria está debilitada por ENM y cifoescoliosis $(1,3)$.

A medida que aumenta la debilidad de la bomba respiratoria, se requiere en forma complementaria al SVNI, manejar la insuficiencia de los músculos espiratorios con técnicas de reclutamiento de volúmenes pulmonares 0 apilamiento de aire (Air Stacking), más tos asistida manual o mecánica con un dispositivo de tos asistida 0 in-exsufflator (MI-E, por su sigla en inglés Mechanical In-exsufflation) $(1,2,3)$. Dichas técnicas permiten permeabilizar las vías respiratorias de las secreciones no movilizadas debido a la debilidad de la tos, de tal manera de prevenir complicaciones asociadas como atelectasias y sobreinfecciones. Estudios recientes han demostrado que los pacientes con ENM avanzadas con CV mínima, tratados con SVNI y MI-E, que generan un pico flujo exuflado máximo con asistencia mecánica de la tos con MI-E (PFE-MI-E) $>150 \mathrm{l} / \mathrm{m}$ pueden evitar la traqueostomía 0 pueden ser decanulados $(10,11,12)$.

Se reportan dos casos clínicos de pacientes con ENM y obesidad ingresados en fecha simultánea, que evolucionaron con insuficiencia respiratoria aguda progresiva, con el objetivo de resaltar las diferencias del manejo convencional con asistencia ventilatoria no invasiva (AVNI) con baja presión de soporte de las terapias respiratorias que combinan el SVNI y la asistencia mecánica de la tos.

\section{CAS0 1.}

Paciente de sexo femenino de 11 años, con antecedente de miastenia gravis y obesidad (peso de 65 $\mathrm{kg}$ ), con anticuerpos anti receptores de acetilcolina positivos y refractaria al tratamiento con prednisona $60 \mathrm{mg}$ al día, piridostigmina $60 \mathrm{mg}$ cada $4 \mathrm{~h}$ y micofenolato mofetil $500 \mathrm{mg}$ cada $12 \mathrm{~h}$.

Ingresa al servicio de Neurología Infantil del Hospital Clínico San Borja Arriarán para evaluación multidisciplinaria y timectomía. Durante la hospitalización evoluciona con infección respiratoria por Metaneumovirus asociada a crisis miasténica que determinó insuficiencia respiratoria aguda progresiva, lo que motivó su traslado a la unidad de cuidados intensivos pediátricos (UCIP). Ingresa con insuficiencia ventilatoria aguda, con acidosis respiratoria con pH 7,25, paCO2 de 55 $\mathrm{mmHg}$ y disfunción de los músculos bulbares con disfagia para alimentos de consistencia líquida y semisólida (papillas), instalándose sonda nasogástrica para su alimentación. La radiografía de tórax mostró atelectasias bibasales. Se indica AVNI con mascarilla nasal y generador de flujo en 2 niveles, BIPAP Trilogy $200 \AA$ en modalidad A/C (asistida/controlada), con volumen promedio asegurado en presión de soporte (AVAPS), con volumen corriente (VC) de $6 \mathrm{ml}$ por kilo de peso $(400 \mathrm{ml})$, presión inspiratoria máxima (IPAPmax) de $20 \mathrm{cmH} 20$, IPAP mínimo (IPAPmin) de $16 \mathrm{cmH} 20$, presión espiratoria (EPAP) de $8 \mathrm{cmH} 20$ y FI02 que se aumenta progresivamente hasta 0,45, para lograr Sp02> 93\% en monitorización no invasiva y control de gases venosos con $\mathrm{Pc} 02<60 \mathrm{mmHg}$. Se realizan además maniobras de tos asistida con ambu-bag, válvula unidireccional y mascarilla nasobucal en ciclos de 4 a 5 por 3 veces al día. Recibe Gamaglobulina endovenosa $2 \mathrm{~g} /$ $\mathrm{kg}$ fraccionada en 5 días consecutivos. Al quinto día en UCIP se traslada a la Unidad de Intermedio (UTIP) con los mismos parámetros de AVNI. Destaca la dificultad respiratoria con $\mathrm{Sp} 02<90 \%$ pese a Fi02 0.4, FR > 30 y murmullo pulmonar muy apagado. Se carece de registro de $\mathrm{CO} 2$ no invasiva por oxicapnografía o C02 transcutáneo (PTCO2). La CV al ingreso a la unidad de intermedio fue de $800 \mathrm{ml}(11 \mathrm{ml} / \mathrm{k})$ y PFT (flujo pico tosido) de 100 LPM. Se decide cambiar la modalidad de la ventilación no invasiva a SVNI con modalidad S/T (espontánea/ tiempo) presión control con Bipap+AVAPS, manteniendo equipo Trilogy $200 \AA$, pero aumentando el VC a $800 \mathrm{ml}$, IPAPmax de 25cmH20, IPAPmin de $18 \mathrm{cmH} 20$ y disminuyendo al mínimo la EPAP a $4 \mathrm{cmH} 20$, para aumentar la entrega de presión diferencial (presión de soporte), bajando progresivamente la Fl02 hasta dejar con aire ambiental. Se agrega rehabilitación respiratoria con protocolo de tos asistida mecánica con in-exsufflator en forma automática, 4 a 5 ciclos con presión inspiratoria de 50 a $60 \mathrm{cmH} 20$, Ti 2 a 3 segundos, con pausa de 1 segundo y presión espiratoria de -50 a $-60 \mathrm{cmH} 20$ con Te de 3 a 4 segundos, con presión manual toracoabdominal durante la fase espiratoria, todas las veces que fuera necesario hasta lograr Sp02 estable > $94 \%$ sin oxigenoterapia. En el seguimiento se observó mejoría clínica, disminución de la FR a rangos normales y mejoría diaria de la CV y PTF.

Al tercer día con CV de $1300 \mathrm{ml}(19 \mathrm{ml} / \mathrm{k})$ y PFT $180 \mathrm{l} / \mathrm{m}$, se inicia ventanas bien toleradas de 1 hora del SVNI, durante alimentación, kinesioterapia motora y deambulación. Al quinto día con una CV de $2600 \mathrm{ml}$ se suspende SVNI durante el día y se mantiene nocturno con los mismos parámetros más tos asistida mecánica 3 veces al día. El día 6 con una CV de $3300 \mathrm{ml}$ y PFT $280 \mathrm{l} / \mathrm{m}$, clínicamente sin dificultad respiratoria, auscultación con ventilación de ambos campos pulmonares hasta las bases y radiografías de control con reexpansión progresiva de atelectasias, se suspende SVNI y se mantiene con rehabilitación pulmonar y motora. Se realiza espirometría que mide una capacidad vital forzada (CVF) de $2490 \mathrm{ml}, 102 \%$ del valor predicho. Se realizan pruebas de medición de presión inspiratoria y espiratoria máxima de oclusión en boca, Pimax-95 cmH20 y Pemax +140 cmH20. Actualmente en espera de realizar timectomía para manejo de patología de base. 


\section{CASO 2.}

Adolescente de 14 años con distrofia muscular de Duchenne (DMD) y obesidad (80 kg de peso), cifoescoliosis severa toracolumbar (600 de angulación) no operada y sin adaptación a sedente en silla de ruedas. Tratado hasta los 10 años con prednisona. Sin colegiatura y con retardo mental leve. Manejado con AVNI desde los 12 años una vez que su CV fue menor del 40\%. Usa Bipap A40® con mascarilla nasal en modo S/T, con IPAP de $12 \mathrm{~cm}$ de H2O, EPAP de $6 \mathrm{~cm}$ de $\mathrm{H} 20$, FR 12, Ti 1.1 seg y tiempo de transición (de subida) de 100 $\mathrm{ms}$.

Ingresa al servicio de pediatría por insuficiencia respiratoria aguda, con taquicardia: $120 \mathrm{lpm}$, polipnea: $35 \mathrm{rpm}$, saturando 85 - $86 \%$ con aire ambiental, febril 38,5 OC y apagamiento del murmullo pulmonar en ambas bases más signología de condensación en la región basal posterior izquierda. Deglución difícil de líquidos y papilla y voz muy apagada. Leucocitosis de 20.000 con predominio de polimorfonucleares y PCR $2,3 \mathrm{mg} / \mathrm{dl}$. Panel viral por inmunofluorescencia identifica Metaneumovirus. La radiografía de tórax destaca severa cifoescoliosis toracolumbar con convexidad derecha, infiltrados intersticiales bilaterales más atelectasias bibasales. Recibe inicialmente hidratación endovenosa, tratamiento con salbutamol en inhalador de dosis medida (MDI). A las 48 h, por aumento de PCR a $39 \mathrm{mg} / \mathrm{dl}$ se agrega ampicilina/sulbactam. Recibió AVNI a través de mascarilla nasobucal con su mismo equipo y parámetros. No se controlan gases sanguíneos y se aumenta oxigenoterapia al circuito de Bipap hasta 7 LPM para objetivo de Sp02> 93\%. Se reevalúa a las 12 horas de su ingreso destacando somnolencia, FR 40 rpm, taquicardia sinusal de 140 Ipm, murmullo pulmonar severamente disminuido y Sp02 de 88-90\% con los parámetros de AVNI ya comentados. La medición de su CV fue de 200 $\mathrm{ml}$, PFT $80 \mathrm{l} / \mathrm{m}$ y PFE-MI-E de $150 \mathrm{l} / \mathrm{m}$.

Se suspenden las inhalaciones de salbutamol y se trata enérgicamente las primeras 2 horas con tos asistida mecánica con in- exsufflator $E 70 \circledR$ de Philips en forma automática, 4 a 5 ciclos con presión inspiratoria de $+50 \mathrm{a}+60 \mathrm{~cm} \mathrm{H} 20$, Ti 2 a 3 segundos, con pausa de 1 segundo y presión espiratoria de - 50 a $-60 \mathrm{~cm}$ H2O con Te de 3 a 4 segundos, hasta lograr Sp02 estable $>94 \%$ sin oxigenoterapia (Figura 1). Para luego pasar a modalidad automática con gatillo por tos (cough track). Se pasa a SVNI con el mismo equipo A40®en modalidad S/T, AVAPS con VC de $1200 \mathrm{ml}$, bajando EPAP al mínimo posible a $4 \mathrm{~cm} \mathrm{H20,} \mathrm{IPAPmax} \mathrm{de} 25 \mathrm{~cm} \mathrm{H} 20$ elPAPmin de $20 \mathrm{~cm} \mathrm{H20}$. Disminuyendo progresivamente la oxigenoterapia hasta suspenderla. Se entrena a la familia en la realización de las maniobras de tos asistida con presión manual toracoabdominal durante la fase espiratoria, utilizando como feedback una Sp02 < de 94\%. Se mantiene con SVNI continuo, presentando mejoría en la auscultación pulmonar, normalización de la FC y FR. Al cuarto día las mediciones de la CV fluctuaban en $200 \mathrm{ml}$, PFT $80 \mathrm{l} / \mathrm{m}$ y PFE-MIE de $200 \mathrm{l} / \mathrm{m}$. Egresa con SVNI continua y tos asistida con programación señalada al séptimo día.

Las características basales de ambos pacientes y su evolución funcional durante el tratamiento con SVNI y tos asistida mecánica se muestran en las tablas 1, 2 y 3 y en las figuras 2 y 3 .

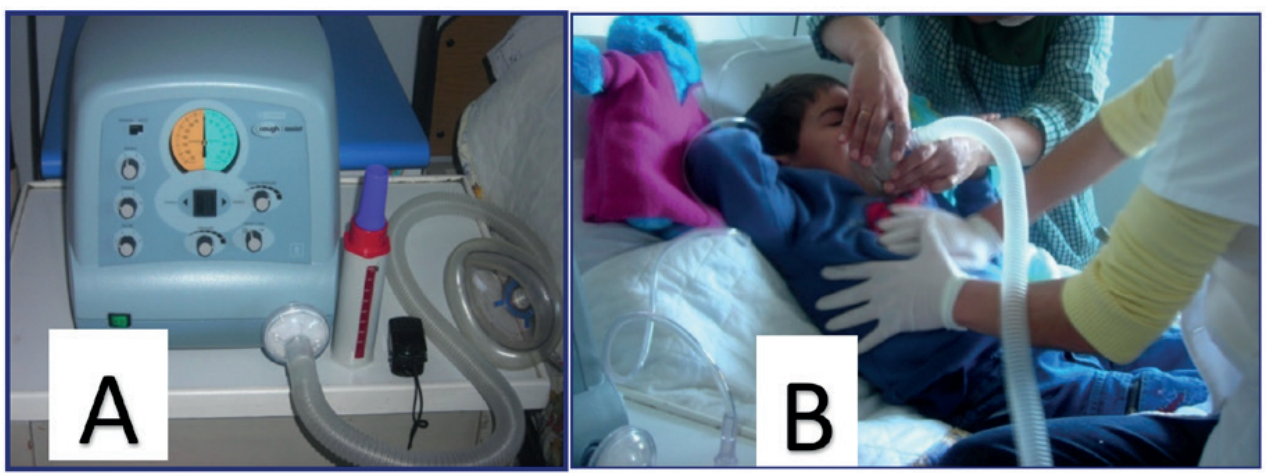

Figura 1. Dispositivos de tos asistida mecánica: A: In- Exsufflator ${ }^{\circledR}$ de Philips, B: maniobra a 4 manos con compresión toraco-abdominal, C: modalidad manual para ajuste de parámetros, D: In- Exsufflator $®$ de Philips, Modelo E70. Mide Pico flujo exhalado máximo con asistencia mecánica de la tos (PFE-MIE).
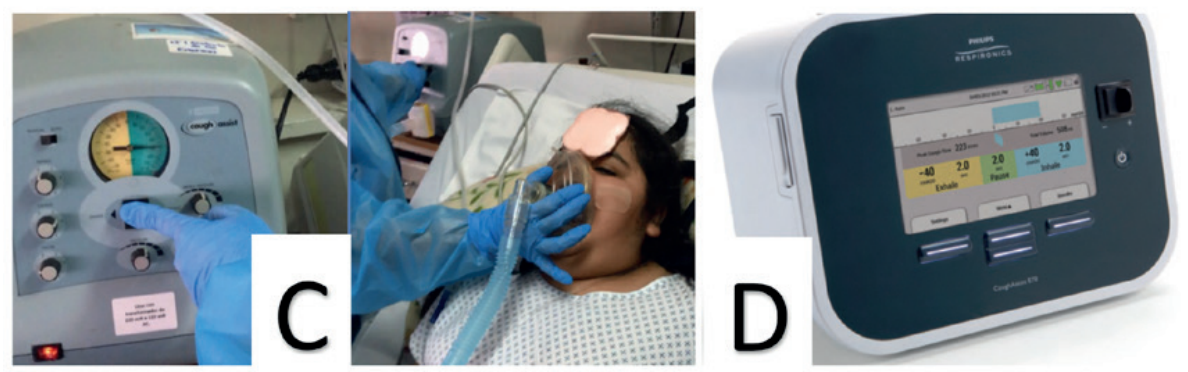
Figura 2. Evolución capacidad vital durante el tratamiento con soporte ventilatorio no invasivo y tos asistida mecánica.

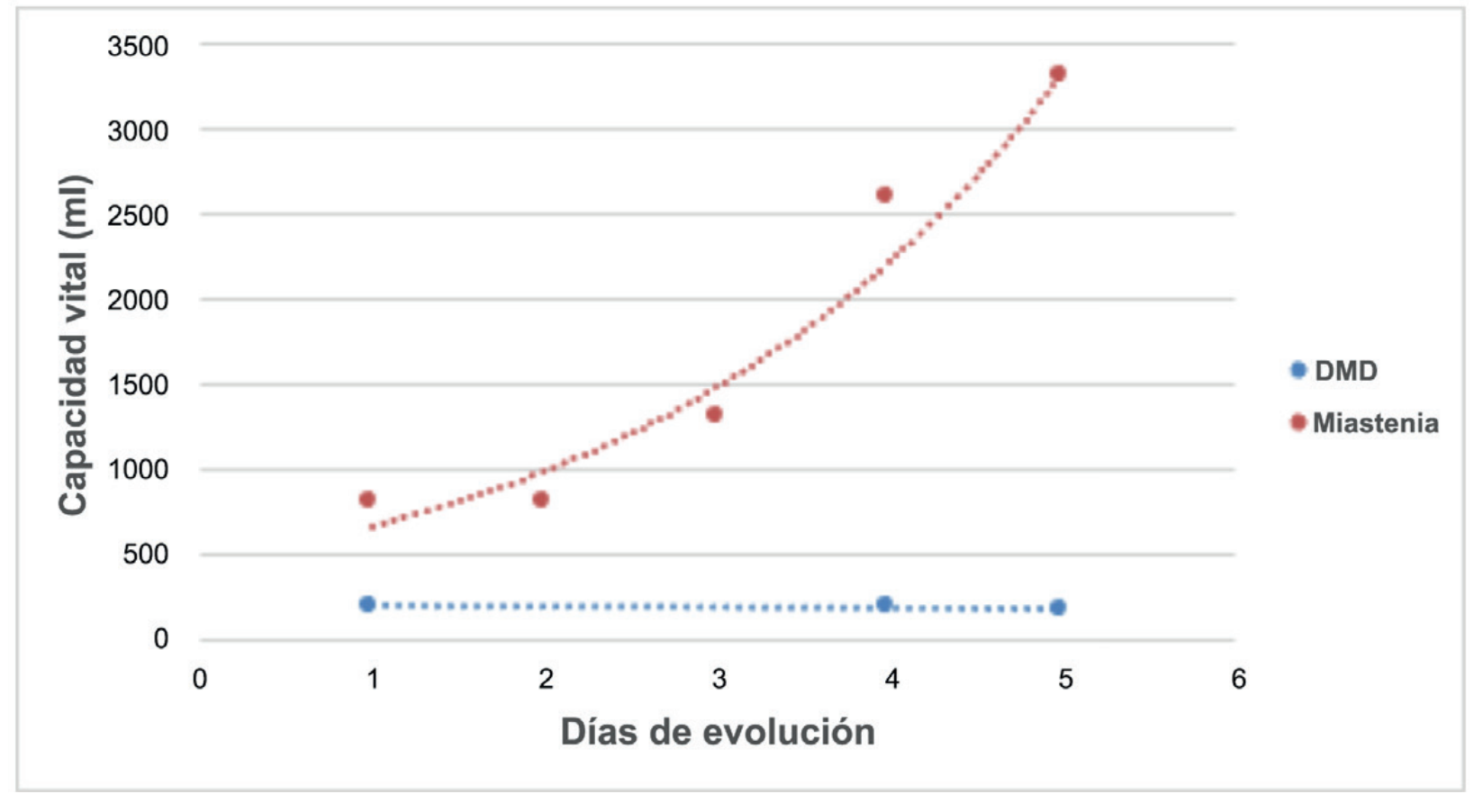

Figura 3. Evolución pico flujo tosido (PFT) y Pico flujo exhalado máximo con asistencia mecánica de la tos (PFE-MIE).

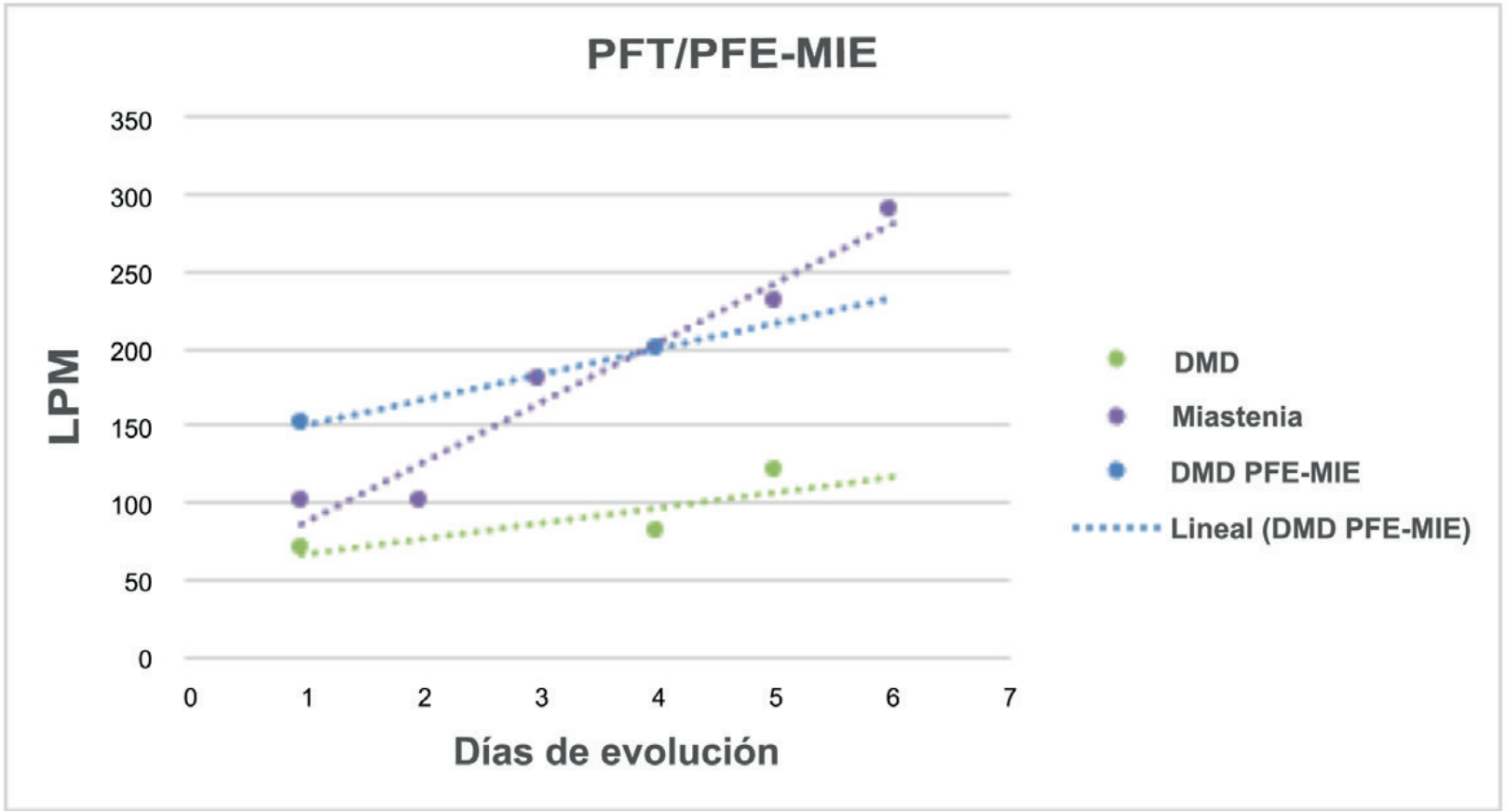

PFT: pico flujo tosido, PFE-MIE: pico flujo exuflado máximo con asistencia mecánica de la tos. (No se presenta en paciente con Miastenia, equipo tos asistida sin flujómetro), LPM: litros por minuto. DMD: distrofia muscular Duchenne. 
Figura 4. Ventilador Trilogy $®$, Philips. A: exhalación pasiva, circuito monorrama, B: exhalación activa, válvula exhalatoria externa, C: Imagen de la válvula exhalatoria y conector en T, D: paciente ventilado con pieza bucal con circuito monorrama.
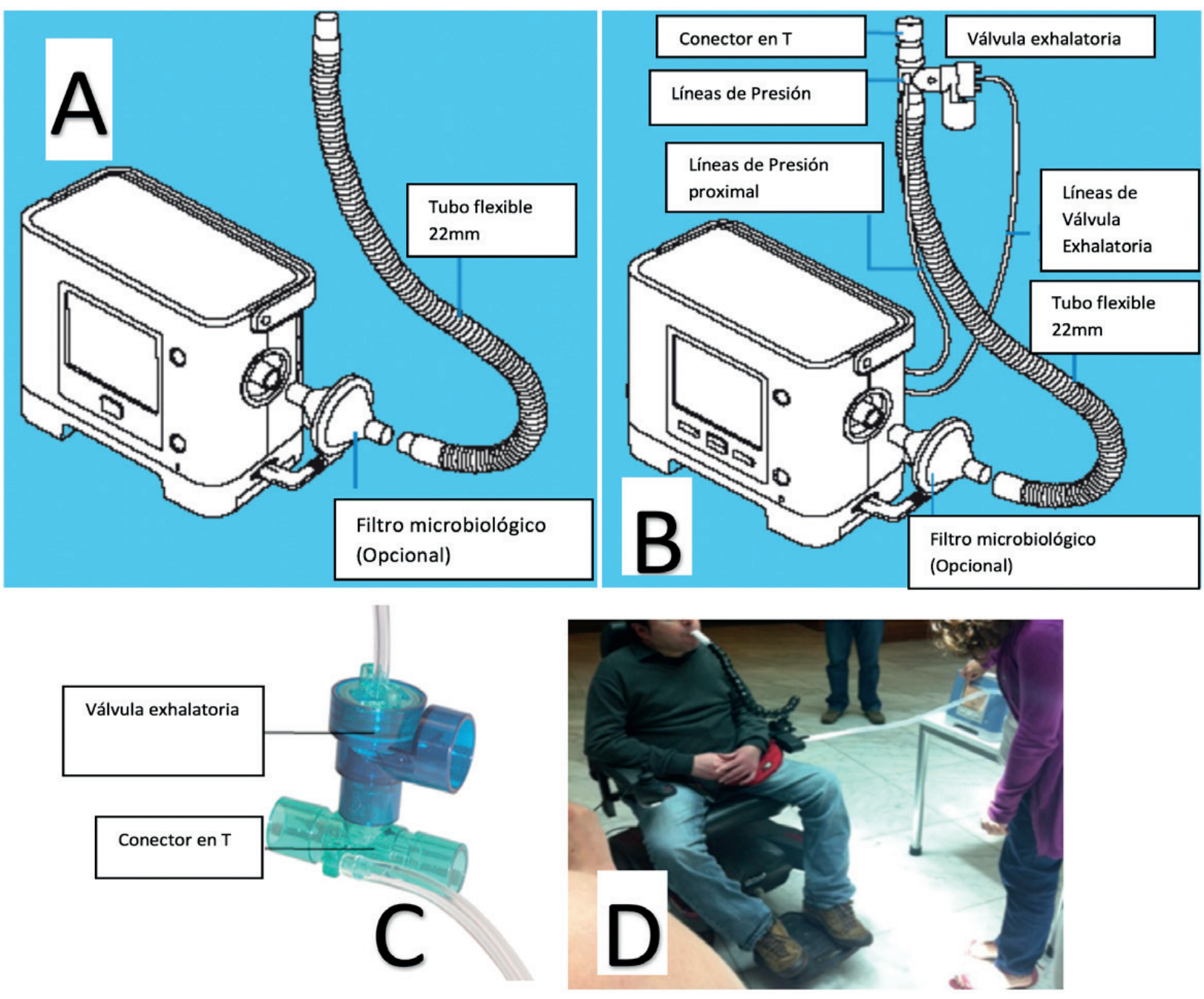

Tabla 1. Características clínicas, funcionales y de tratamiento al ingreso de ambos pacientes.

\begin{tabular}{|c|c|c|c|c|c|c|c|c|c|c|c|c|}
\hline Paciente & Sexo & Edad & Peso & $\begin{array}{l}\text { Patología } \\
\text { asociada }\end{array}$ & TTO previo & $\begin{array}{l}\text { Causa } \\
\text { ingreso }\end{array}$ & Causa IR & $\begin{array}{l}\text { Presencia } \\
\text { Atelectasia }\end{array}$ & $\begin{array}{l}\text { Presencia } \\
\text { Neumonía }\end{array}$ & $\mathrm{PaCO}_{2} / \mathrm{SpO}_{2}$ & $\begin{array}{l}\text { CV al } \\
\text { ingreso SVNI }\end{array}$ & $\begin{array}{l}\text { PFT al } \\
\text { Ingreso SVNI }\end{array}$ \\
\hline Miastenia & $\mathrm{F}$ & 11 & $65 \mathrm{~K}$ & Obesidad & $\begin{array}{l}\text { Prednisona/ } \\
\text { Piridostigmina/ } \\
\text { Micofenolato }\end{array}$ & Timectomia & $\begin{array}{l}\text { Infección } \\
\text { respiratoria } \\
\text { viral/Crisis } \\
\text { Miasténica }\end{array}$ & Bibasal & No & $\begin{array}{l}55 \mathrm{mmHg} / \\
89-90 \%\end{array}$ & $800 \mathrm{ml}$ & $100 \mathrm{l} / \mathrm{m}$ \\
\hline DMD & M & 14 & $80 \mathrm{~K}$ & $\begin{array}{l}\text { Obesidad. } \\
\text { Cifoscoliosis } \\
\text { Angulo } \\
\text { Cobb } 60^{\circ}\end{array}$ & AVNI noche & Artrodesis & $\begin{array}{l}\text { Infección } \\
\text { respiratoria } \\
\text { viral }\end{array}$ & Bibasal & $\begin{array}{l}\text { Basal } \\
\text { izquierda }\end{array}$ & $\begin{array}{l}43 \mathrm{mmHg} / \\
85-86 \%\end{array}$ & $200 \mathrm{ml}$ & $80 \mathrm{l} / \mathrm{m}$ \\
\hline
\end{tabular}

DMD: distrofia muscular de Duchenne, AVNI: asistencia ventilatoria no invasiva, IR: insuficiencia respiratoria, SVNI: soporte ventilatorio no invasivo, PaCo2: presión arterial de Co2, Sp02: saturación de oxígeno, PFT: flujo pico tosido. 
Tabla 2. Características de las terapias ventilatorias en ambos pacientes: AVNI (Asistencia Ventilatoria No Invasiva con generador de flujo en 2 niveles) y SVNI (Soporte Ventilatorio No Invasivo).
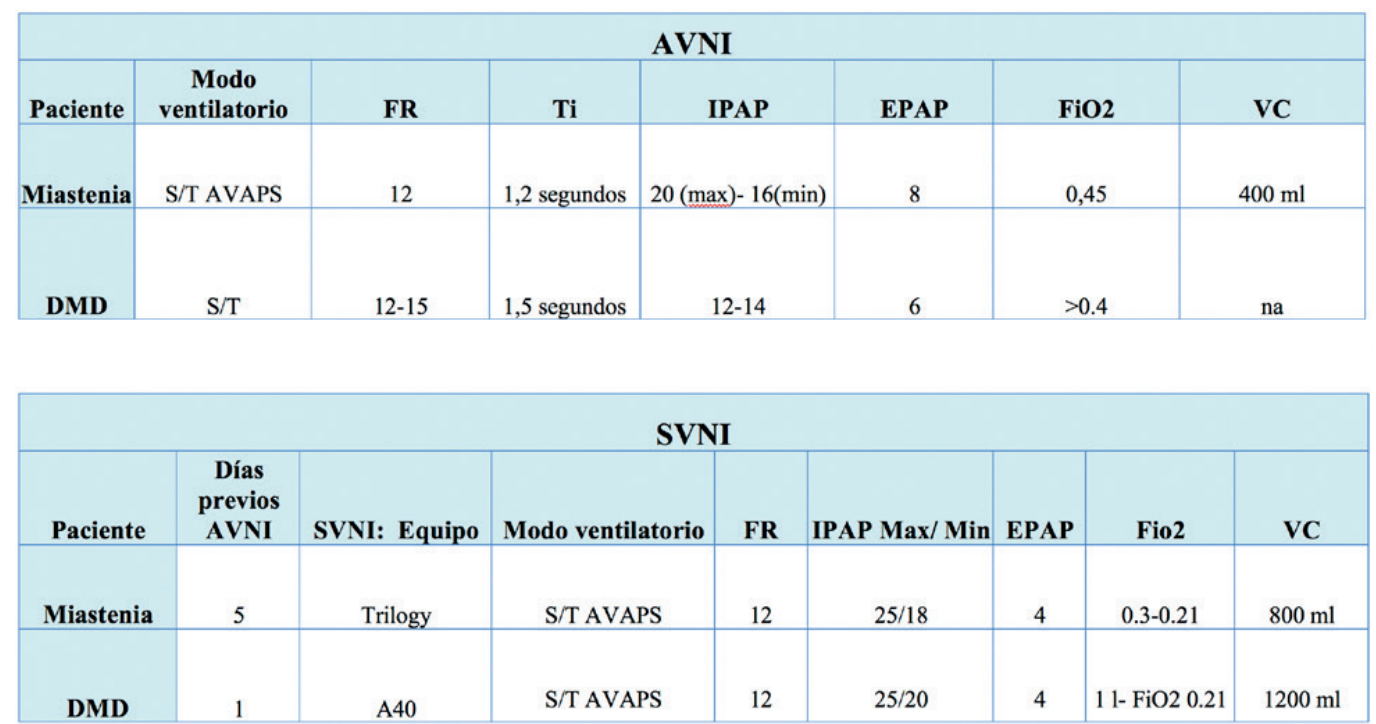

AVAPS: Volumen promedio asegurado en presión de soporte, IPAP: presión inspiratoria, EPAP: presión espiratoria, Fi02: fracción inspirada de oxígeno, S/T: modalidad espontanea/ tiempo, FR: frecuencia respiratoria, Ti: tiempo inspiratorio, Te: tiempo espiratorio, PaC02 Presión arterial de Co2, Sp02: Saturación de oxígeno, registro no invasivo, VC: volumen corriente, na: no aplica.

Tabla 3. Evolución durante tratamiento con soporte ventilatorio no invasivo y tos asistida mecánica.

\begin{tabular}{|r|c|c|c|c|c|c|}
\hline \multirow{2}{*}{ Dia } & \multicolumn{3}{|c|}{ Caso 1 (Crisis Miasténica) } & \multicolumn{3}{c|}{ Caso 2 (DMD) } \\
\cline { 2 - 7 } & CV (ml) & PFT (1/m) & PFE MI-E (1/m) & CV (ml) & PFT (1/m) & PFE MI-E (1/m)* \\
\hline $\mathbf{1}$ & 800 & 100 & (n.a.) & 200 & 70 & 150 \\
\hline $\mathbf{2}$ & & & & & & \\
\hline $\mathbf{3}$ & 1300 & 180 & (n.a.) & & & \\
\hline $\mathbf{4}$ & & & & 200 & 80 & 200 \\
\hline $\mathbf{5}$ & 2600 & 230 & (n.a.) & 180 & 120 & \\
\hline $\mathbf{6}$ & 3300 & $290(* 1)$ & (n.a.) & & & \\
\hline
\end{tabular}

DMD: distrofia muscular de Duchenne, CV: capacidad vital, PFT: pico flujo tosido, PFE-MI-E: pico flujo exuflado máximo con in-exsufflator, n.a: no aplica. $\left(^{\star}\right)$ Se mide sólo en equipo E70.

\section{DISCUSIÓN}

Ninguno de los pacientes reportados tenía evaluación funcional respiratoria durante los 12 meses previos al ingreso que permitiera precisar el compromiso de los músculos inspiratorios y espiratorios. Ninguno de ellos reportaba clínica sugerente de compromiso de la musculatura bulbar previo a su agudización, la cual fue gatillada en ambos casos por una infección viral por Metaneumovirus. Ambas patologías corresponden a ENM con presentaciones y cursos evolutivos distintos, en el primer caso con una evolución tórpida de debilidad muscular fluctuante y refractaria a los tratamientos inmunomoduladores con persistencia de anticuerpos anti receptores de acetilcolina, con episodios previos de crisis miasténica y con indicación clara de timectomia $(14,15,16)$ pero con deambulación y vida escolar normal. El paciente con DMD, contrariamente, con una evolución muy avanzada de su enfermedad, con cifoescoliosis severa sin artrodesis y postración. Ambas condiciones asociadas a una importante disminución de la CV y de la función ventilatoria $(1,2,3)$. Tratado, a nuestro entender, insuficientemente con AVNI y diferenciales de presión bajos desde los 12 años $(17,18)$, poco tiempo después de haber perdido la bipedestación y suspendido los corticoides orales, con baja adherencia a Ios protocolos de apilamiento de aire y tos asistida manual con ambu bag (19-25). Ambas estrategias de rehabilitación respiratoria permiten disminuir la velocidad de la pendiente de declinación de la CV, una vez alcanzado su plateau, después de haber perdido la bipedestación(26). 
Ambos pacientes eran obesos (IMC > 40), condición que reconocidamente se asocia con SAOS, y en algunos de ellos, a síndrome de hipoventilación por distintos mecanismos fisiopatológicos, como sobrecarga de la musculatura, insuficiencia de los músculos de la bomba respiratoria, defectos del centro respiratorio y de su sensibilidad a la hipercapnia (9), que en pacientes con ENM, necesariamente condiciona un mayor compromiso de la bomba respiratoria.

Es habitual, que pacientes con ENM y exacerbaciones por infecciones respiratorias banales, evolucionen con insuficiencia ventilatoria. En nuestros pacientes por causalidades distintas; gatillando una crisis miasténica y en el paciente con DMD descompensación de una insuficiencia ventilatoria crónica.

En la insuficiencia respiratoria aguda con pulmón enfermo e hipoxemia secundaria a trastorno de ventilación/ perfusión (V/Q) el tratamiento es la oxigenoterapia. En aquellos que requieren asistencia ventilatoria por insuficiencia respiratoria hipercápnica, tradicionalmente se utiliza AVNI, generalmente con presión diferencial baja en un símil al concepto de ventilación protectora con VC de $6-8 \mathrm{ml} / \mathrm{k}$, utilizada en los pacientes intubados y con síndrome de distrés respiratorio agudo (SDRA). Utilizar estos parámetros en pacientes con insuficiencia ventilatoria secundaria al compromiso de los músculos respiratorios por ENM y obesidad es un error frecuente $(1,2,3)$. El uso de oxigenoterapia con o sin AVNI convencional pone a estos pacientes en riesgo de hipoventilación progresiva e hipercapnia severa, la que de no reconocerse, aumenta el riesgo de intubación, de falla de extubación una vez ventilados en forma invasiva, y en consecuencia, también de una indicación innecesaria de traqueostomía (27). Peor aún y más grave, los pacientes con debilidad de los músculos espiratorios y compromiso bulbar con hipercapnia progresiva pesquisados tardíamente, pueden presentar paro cardiorespiratorio y muerte (28).

En pacientes con MG, los predictores de un mayor riesgo de intubación endotraqueal para ventilación mecánica invasiva son la < CV (bajo $15 \mathrm{ml} / \mathrm{k}$ ), la severidad de la hipercapnia, la intensidad del compromiso de la musculatura bulbar y la capacidad tusígena (29). Pese a la utilización de AVNI convencional, en aquellos pacientes con $\mathrm{PaC0} 2<60 \mathrm{mmHg}$, se logra prevenir la intubación en casi $2 / 3$ de ellos (29). En pacientes con ENM tan graves como la atrofia muscular espinal (AME) tipo 1 y en aquellos con AME tipo 2 ó DMD avanzada y con CV $<10 \mathrm{ml} / \mathrm{k}$, es posible mantenerlos por largo tiempo en SVNI incluso continuo, evitando la traqueostomía, y tratar apropiadamente las agudizaciones con un bajo riesgo de intubación, siendo posible decanularlos cuando han sido traqueostomizados por necesidad de ventilación mecánica prolongada (3033). Incluso pese a tener severo deterioro de la función de los músculos bulbares y trastornos de la deglución que los obliguen a ser gastrostomizados precozmente, a diferencia de los pacientes con enfermedades del SNC y compromiso bulbar por afectación de la motoneurona superior, que condiciona espasticidad de los músculos faríngeos que impide la generación de flujos exuflados máximos con MI-E (PFE-MIE) >150 l/m (10).

Ambos pacientes reportados, corresponden a pacientes portadores de ENM sin compromiso bulbar por enfermedades del SNC y por lo tanto pese al severo deterioro funcional con CV casi 0 del paciente con DMD y menor de $15 \mathrm{ml} / \mathrm{kilo}$ en la paciente con crisis miasténica y trastorno de la deglución secundario, fue posible obtener flujos exhalados, PFE-MIE y PEF respectivamente por arriba de los límites de riesgo para intubación endotraqueal, Io que permitió efectivamente limpiar las vías aéreas de secreciones, evitando la ventilación mecánica invasiva. Incluso en el paciente con DMD cuya CV no mejoró.

A diferencia de la AVNI, que generalmente es entregada con generadores de flujos capaces de dar presiones en 2 niveles y programada con presión de soporte 0 diferencial entre la IPAP y EPAP $<$ de $15 \mathrm{~cm}$ de $\mathrm{H} 20$, con $\mathrm{VC}<10 \mathrm{ml}$ por kilo y EPAP $>4 \mathrm{~cm}$ de $\mathrm{H} 2 \mathrm{O}$, el SVNI puede ser entregado con ventiladores convencionales con exhalación activa a través de una válvula exhalatoria que se cierra durante la espiración y permite presurizar la vía aérea en modalidad $\mathrm{A} / \mathrm{C}$ por volumen control con 12 a $15 \mathrm{ml}$ por kilo y en niños mayores de 10 años con VC de $1000-1500 \mathrm{ml}$ y Ti de 1,5 segundos y frecuencias respiratorias de 10 a 15 RPM. Se pueden utilizar distintas interfaces como cánulas nasales, prongs 0 almohadillas, mascarillas nasales y piezas bucales anguladas de 15 $\mathrm{mm}$. En esta modalidad ventilatoria es posible entregar PEEP $=0$, de tal modo que la presión inspiratoria no sea menor de 15 - $18 \mathrm{~cm}$ de H20, constituyendo diferenciales de presión suficientes para lograr una ventilación alveolar apropiada $(1,2,3)$. En nuestros pacientes utilizamos una variante novedosa de SVNI con ventiladores híbridos ciclados por flujo y volumen garantizado (AVAPS) (Figura 4), con flujos inspiratorios acelerados desde un nivel basal de presión no menor de $4 \mathrm{~cm}$ de H2O hasta 2 niveles distintos de IPAP (máximo y mínimo), que permita entregar un VC objetivo > de $10 \mathrm{ml} / \mathrm{k}$ (2). Esto a través de un circuito monorrama con concepto de exhalación pasiva con un flujo de escape intencional en puntos exhalatorios adhoc en las mascarillas nasales (2). Con esto se logró una rápida mejoría clínica con normalización de la FR y Sp02 estables sin suplementación de oxigenoterapia, como es recomendado en pacientes con ENM $(1,2,3,26)$. Además de mejoría en la CV medida con ventilómetro Wright de Ferrari® y una pieza bucal en la paciente miasténica.

A diferencia de la AVNI convencional que puede eventualmente no evitar el riesgo de intubación endotraqueal, el SVNI, aún en pacientes con ENM con CV muy bajas, como en los 2 pacientes reportados, junto con el uso de protocolos de tos asistida mecánica (M-IE) son efectivos para tratar la insuficiencia ventilatoria grave y deben constituirse como el manejo respiratorio no invasivo estándar (1,2,3,21-25,28,30,32,33).

\section{CONCLUSIÓN}

El SVNI/M-IE evitan la intubación endotraqueal en pacientes con diagnóstico de ENM y obesidad que están cursando insuficiencia respiratoria aguda, a diferencia del agravamiento de la misma que produce la AVNI y oxigenoterapia con criterios clásicos.

\section{Los autores declaran no tener conflictos de interés.}




\section{REFERENCIAS}

1. Bach JR. Noninvasive Respiratory Management of Patients with Neuromuscular Disease. Ann RehabilMed 2017; 41:519-538.

2. 2. Prado F., Salinas P., Pinchak C., et al. Cuidados Respiratorio para Pacientes con Enfermedades Neuromusculares. NeumolPediatr 2017; 12 (3): 103 113.

3. Bach JR., Mehta AD. Respiratory muscle aids to avert respiratory failure and tracheostomy: a new patient management paradigm. Journal of Neurorestoratalogy2014; 2:25-35.

4. Bach JR., Sabharwal S. High Pulmonary Risk Scoliosis Surgery Role of Noninvasive Ventilation and Related Techniques. Spinal Disord Tech 2005; 18:527-530.

5. Gill I., Eagle M., Mehta JS.,et al. Correction of neuromuscular scoliosis in patients with preexisting respiratory failure. Spine 2006; 31: 2478-2483.

6. Pavo J., Perez-Grueso FS., Fernandez-BailloN.,et al.Severe restrictive lung disease and vertebral surgery in pediatric population. EurSpine J 2009; 18: 19051910.

7. Takaso M., Nakazawa T., Imura T., et al. Surgical management of severe scoliosis with high-risk pulmonary dysfunction in Duchenne muscular dystrophy. IntOrthop 2010; 34: 401-406.

8. Prado F., Salinas P., García C. Recomendaciones para la evaluación quirúrgica de la escoliosis en niños con enfermedad neuromuscular. NeumolPediatr 2010; 5: 67 $-73$.

9. Branstiter G., Lempert N., Sotomayor T., et al. Obesity hypoventilation syndrome in a 12-year-old child requiring therapeutic phlebotomy: Case report and review of the literatura. PediatrInt. 2014; 56: 99-101.

10. Bach JR., Giménez C. Mechanical In-exsufflationExpiratory Flows as Indication for Tracheostomy Tube Decannulation: Case Studies. Am J Phys Med Rehabil 2019; 98: 18-20.

11. Suarez A., Pessolano F., MonteiroS., et al. Peak flow and peak cough flow in the evaluation of expiratory muscle weakness and bulbar impairment in patients with neuromuscular disease. Am J Phys Med Rehabil.2002; 81:506-11.

12.

13. Kang SW., Bach JR. Maximum insufflation capacity: vital capacity and cough flows in neuromuscular disease. Am J PhysMedRehabil2000; 79:222-227.

14. Fernández JA., Fernández-Valiñas A., Aldrete-Velasco J.CrisisMiasténica. MedlntMéx. 2016; 32:341-354.

15. G. Morís. Miastenia gravisy trastornos relacionados con la unión neuromuscular. Medicine2019; 12:4469-77.

16. MartínezS., Gómez M., Martínez R. Anupdateonmyastheniagravis. Semergen. 2018; 44:351-354

17. Andrews JG., Soim A., Pandya S., et al. Respiratory Care Received by Individuals withDuchenne Muscular Dystrophy From 2000 to 2011. Respir Care. 2016; 61:1349-59.

18. Gonçalves MR., Bach JR., Ishikawa Y., et al. Continuous noninvasive ventilatory support outcomes for neuromuscular disease: a multicenter collaboration and literature review. European Respiratory Review 2016;25.

19. Bach JR., De Cicco A. Forty-eight years with Duchennemuscular dystrophy. Am J Phys Med Rehabil
2011; 90:868- 812.

20. Bach J., Alba A., Pilkington L., et al. Long-term rehabilitation in advanced stage of childhood onset, rapidly progressive muscular dystrophy. Arch Phys Med Rehabil1981; 62:328-331.

21. Toussaint M., Pernet K., Steens M., et al. Cough Augmentation in Subjects withDuchenne Muscular Dystrophy: Comparison of Air Stacking via a Resuscitator Bag Versus Mechanical Ventilation. Respir Care 2016; 61:61- 7.

22. Bach JR., Sinquee D., SaporitoLR., et al.Efficacy of mechanical insufflation-exsufflation in extubatingunweanable subjects with restrictive pulmonary disorders. Respir Care 2015; 60:477-483.

23. Bach JR., Gonçalves MR., Hamdani I., et al.Extubation of unweanable patients with neuromuscular weakness: a new management paradigm. Chest 2010; 137:10331039.

24. Bach JR., Chiou M., Saporito LR.,et al. Evidence- Based Medicine Analysis of mechanical insufflation- Exsufflation Devices. Respir Care2017; 62:643.

25. Bach JR., Saporito LR., Shah HR., et al.Decanulation of patients with severe respiratory muscle insufficiency: efficacy of mechanical insufflation-exsufflation. J Rehabil Med 2014; 46:1037-1041.

26. Chiou M., Bach JR., Jethani L., et al. Active lung volumen recruitment to preserve vital capacity in Duchenne muscular dystrophy. J Reabil Med 2017 19; 49:49-53.

27. Bach JR., Rajaraman R., Ballanger F., et al. Neuromuscular ventilatory insufficiency: the effect of home mechanical ventilator use vs. oxygen therapy on pneumonia and hospitalization rates. Am J Phys Med Rehabil1998; 77:8-19

28. Bach J., Goncalves M., Hon A., et al. Changing trends in the Management of end-stage neuromuscular respiratory muscle failure. Am J Phys Med Rehabil 2013; 92: 266-77.

29. Wu JY.,Kuo PH., Fan PC.,et al The role of noninvasive ventilation and factors predicting extubation outcome in myasthenic crisis. Neurocrit Care. 2009; 10: 35-42.

30. Bach JR., Sinquee D., Saporito LR.,et al. Efficacy of mechanical insufflation-exsufflation in extubatingunweanable subjects with restrictive pulmonary disorders. Respir Care 2015;60:477-483.

31. McKim D., Griller N., LeBlanc C., et al. Twenty- four hour noninvasive ventilation in Duchenne muscular dystrophy: A safe alternative to tracheostomy. Can Respir J 2013; 20:5-9

32. Villanova M.,BrancalionB., MehtaAD.Duchenne muscular dystrophy: life prolongation by noninvasive ventilatory support. Am J Phys Med Rehabil 2014;93:595-597.

33. Bach JR., Martinez D. Duchenne muscular dystrophy: prolongation of survival by noninvasive interventions. RespiratoryCare2011; 56:744 758. 